\title{
EL DESARROLLO DEL PENSAMIENTO CRÍTICO Y SU IMPORTANCIA EN LA FORMACIÓN UNIVERSITARIA
}

\author{
Héctor M. Morán Seminario \\ Universidad Ricardo Palma \\ hemo26@yahoo.es
}

\section{RESUMEN}

En el presente artículo se hace una revisión sobre la pregunta ¿qué es el pensamiento crítico? Se analiza desde diferentes perspectivas teóricas para conocer su significado y su importancia en educación superior; se presentan los principales elementos en la comprensión del pensamiento crítico y sus usos. Se proponen también algunas herramientas para que los docentes fomenten y desarrollen en los estudiantes el pensamiento crítico en las distintas actividades académicas, ya sea al interior del aula o en otros espacios. A su vez, se exponen las habilidades básicas que le son características, haciendo énfasis en la solución de problemas. Finalmente, se proponen algunas estrategias de enseñanza a utilizar en el salón de clases con la finalidad de que los estudiantes universitarios aprendan a pensar críticamente.

\section{PALABRAS CLAVE}

Pensamiento crítico/ Docencia, educación superior/ Desarrollo de habilidades del pensamiento crítico

\begin{abstract}
This paper reviews the question: what is critical thinking? The review is conducted from different theoretical perspectives to understand its meaning and relevance in higher education. It's presents the main elements and their use in the understanding of what is critical thinking. Some tools are also proposed for teachers to promote and develop the critical thinking in the students in the different academic activities, either within the classroom or in other environments.with an emphasis on basic skills used in problem solving. Finally, the paper proposes a set of teaching strategies to help students learn to think critically through the application inclassroom discussion.
\end{abstract}

\section{KEY WORDS}

Critical thinking, Teaching/ Higher education/ Development of critical thinking skills 


\section{INTRODUCCIÓN}

Los años 2015 y el 2016 el diario El Comercio informaba sobre las declaraciones que con gran preocupación el Presidente de la Asociación de Ingenieros del Perú y el Presidente de la CONFIEP lamentaban de que los egresados de las universidades peruanas no acreditaban las competencias que necesitaban las empresas nacionales, entre ellas puntualmente señalaban ambos representantes la falta de "creatividad"; en el caso de los egresados de ingeniería esta falta de originalidad y creatividad los calificaban más como "artesanos" que como auténticos "ingenieros". Ante estas carencias, según manifestaban, los empresarios se ven en la necesidad de contratar profesionales egresados del exterior que llenen sus requerimientos. Declaraciones, pesimistas por cierto, que llevan a preguntar ¿Qué está pasando con la formación de los egresados en nuestras universidades?

Ahora, a nivel macro observamos que dentro de todas las situaciones que afectan la educación en el país una se relaciona con la falta de pensamiento crítico en los estudiantes, lo que tampoco es nuevo descubrimiento. Y ¿cómo se presenta esta misma problemática en las universidades?, por la información recabada podemos afirmar que el problema también es preocupante; los estudiantes por lo regular carecen de esta competencia, por lo que es un campo interesante de investigación. Los proyectos y la literatura sobre esta problemática universitaria no son en nuestros medio muy abundantes aún. Pero el problema está allí, esperando ser investigado. Aunque no existan estadísticas que respalden la existencia del mencionado problema, en nuestra experiencia y el intercambio de información entre docentes es común escuchar afirmaciones como: el alumno no "piensa" ni sabe analizar porque no "desarrolla". Afirmaciones que suenan desproporcionadas que y aunque se puedan disimular pero no negar, como se verá más adelante.

Contribuir al desarrollo intelectual de los estudiantes es un objetivo fundamental de la educación universitaria, al tiempo que un desafío para quienes que acompañamos procesos de formación. El desarrollo del pensamiento crítico es inherente a las acciones para favorecer el rigor intelectual y el aprendizaje autónomo. El pensamiento crítico permite a los estudiantes tratar adecuadamente la creciente información disponible en la sociedad, analizar afirmaciones o creencias para evaluar su precisión, pertinencia o validez y elaborar juicios basados en criterios que consideren el contexto.

¿Qué significa pensar críticamente? ¿Por qué es importante promover el pensamiento crítico en la universidad? ¿Qué ventajas aporta para la formación de 
los futuros profesionistas? ¿Por qué deben los docentes universitarios desarrollarlo en sus estudiantes? Éstas y muchas otras preguntas surgen cuando nos detenemos a reflexionar sobre el significado de pensar críticamente.

Nuestra tesis es que nuestro sistema educativo se ha orientado a entrenarnos para repetir la información que se considera relevante para comprender el mundo, más que a cuestionarla. Pues toda educación tiene una indispensable función conservadora, quizá la de mayor peso, gracias a la cual logra consolidarse nuestra civilización. De ahí que cuando miramos las cosas de este modo parecería que el pensamiento crítico es un arma peligrosa que amenaza con regresarnos atrás en el camino del conocimiento humano. Muchas veces dudar, cuestionar, poner en tela de juicio, mostrarse escéptico ante ciertas afirmaciones, no es signo de inteligencia, sino de profunda excentricidad sobre algún asunto. Entonces, ¿Cómo y cuándo es válido cuestionar? ¿De qué manera debe insertarse el pensamiento crítico en la educación de la juventud? Estas preguntas nos remiten a una más básica: ¿Qué entendemos por pensamiento crítico? En las siguientes secciones Intentaremos responderlas, plausiblemente.

\section{¿QUÉ ES EL PENSAMIENTO CRÍTICO?}

En las últimas décadas uno de los principales objetivos de las instituciones de educación superior en el mundo ha sido desarrollar el pensamiento crítico en los estudiantes, es decir, formar alumnos capaces de argumentar sus ideas, evaluar lo expuesto por los otros y razonar con rigor científico en cualquier asignatura curricular, pero fundamentalmente capaces de tomar decisiones sólidas y resolver problemas de manera eficaz. Muchos proyectos educativos particulares señalan abiertamente que la formación de los estudiantes universitarios debe estar orientada a desarrollar habilidades reflexivas, críticas y de investigación que fortalezcan su voluntad de indagar y conocer.

Igualmente, el pensamiento crítico ha recibido atención de la Organización de las Naciones Unidas para la Educación, la Ciencia y la Cultura (Unesco) en la Declaración Mundial sobre la Educación Superior en el Siglo xxi. Visión y Acción y en el Marco de Acción Prioritaria para el Cambio y el Desarrollo de la Educación Superior, formulados en 1998 y ratificados en 2003 en la Conferencia Mundial sobre la Educación Superior (Cmes), destacando la importancia de formar estudiantes con un pensamiento crítico, capaces de analizar los problemas de la sociedad, buscar soluciones conjuntamente con su aplicación y asumir responsabilidades sociales. 
Ahora bien, pensar críticamente implica saber utilizar la información disponible: buscarla, cuestionarse sobre ella, pero principalmente ser capaz de elaborar, poco a poco, una postura personal en diálogo constante con los datos y los autores. Es más una actitud de vida que una habilidad aislada, pues implica al sujeto integral, con razón y con emoción, con deseos y voluntad (Patiño, 2012).

Por su parte Peter Facione, (citado por Patiño (2012, p. 3) ofrece la definición de pensamiento crítico consensada por la Asociación Filosófica Americana:

"Entendemos que el pensamiento crítico (pc) es el juicio autorregulado y con propósito que da como resultado interpretación, análisis, evaluación e inferencia, como también la explicación de las consideraciones de evidencia, conceptuales, metodológicas, criteriológicas o contextuales en las cuales se basa ese juicio. El pc es fundamental como instrumento de investigación. Como tal, constituye una fuerza liberadora en la educación y un recurso poderoso en la vida personal y cívica de cada uno. Si bien no es sinónimo de buen pensamiento, el pc es un fenómeno humano penetrante, que permite autorrectificar" (Facione, 2007, 21).

Si tratamos de identificar los rasgos del pensamiento crítico es probable que coincidamos en los siguientes propuestos de Facione:

Examinar ideas: Significa identificar el papel que juegan varias expresiones en el contexto de una argumentación; ser capaz de comparar y contrastar ideas, identificar puntos de controversia y determinar las partes que los componen; de identificar las relaciones de las partes con el todo de un argumento.

Identificar argumentos: Implica detectar la tesis central de un argumento $\mathrm{y}$, con ello, las razones que apoyan o contradicen ese argumento o punto de vista.

Analizar argumentos: De lo que se trata es de identificar y diferenciar:

a) La conclusión principal,

b) Las premisas y razones que se presentan para apoyar la conclusión principal,

c) Las premisas y razones adicionales que se presentan como apoyo de aquellas premisas y razones,

d) Los elementos adicionales del razonamiento que no se presentan explícitamente, como conclusiones intermedias, suposiciones 0 presupuestos,

e) La estructura general del argumento o hilo de razonamiento, 
f) Los elementos que hacen parte de lo que se está examinando pero que no son parte del razonamiento: el contexto o el telón de fondo del mismo.

Evaluar argumentos: Es una actividad que generalmente se realiza a la par que se identifica el argumento. La evaluación es la "valoración de la credibilidad de los enunciados o de otras representaciones que recuentan o describen la percepción, experiencia, situación, juicio, creencia u opinión de una persona " (Patiño, 2012, $8-9)$.

Por lo expresado hasta aquí, en la educación superior se acentúa la importancia de formar estudiantes capaces de descubrir, generar y aplicar nuevos conocimientos en situaciones cambiantes, de buscar soluciones novedosas y eficaces a los problemas y retos con los que se enfrentan, de actuar con confianza y decisión ante las exigencias del porvenir.

Para conocerlo más a fondo, haremos una revisión sucinta de los diversos enfoques en torno al tema para comprender su significado y conocer cómo se ha promovido.

\section{DEFINICIONES Y CONCEPTUALIZACIONES DE PENSAMIENTO CRÍTICO}

Seguiremos en este apartado a Patricia Alvarado, pues ella considera que el pensamiento crítico es un concepto clave por su importancia para que los estudiantes logren aprender a aprender, que es uno de los cuatro pilares de la educación. Y que por su complejidad, no hay una definición unánime, y ha sido definido e interpretado de muchas maneras por diversos autores, ha viendo reunido una interesante y relevante síntesis sobre el particular que utilizamos para nuestros propósitos. Así, según Pintrich y García, citados por Javier Ignacio Montoya (2007), el pensamiento crítico es ante todo "una estrategia cognitiva, que se refiere al intento de los estudiantes de pensar de un modo más profundo, reflexivo y cuestionador el material de estudio". Para Paul (2000), en coincidencia con Norris y Ennis (1989), es el proceso de reflexión y discriminación entre diversas perspectivas, propias y ajenas para determinar cuál es la "verdad" que cada individuo juzga conveniente aceptar para la resolución de problemas. Que en opinión de Johnson (2008), es la teoría de la argumentación, entendida como el saber que buscamos, donde se abordan problemas o se plantean objetivos que se intentan resolver o alcanzar. Otro enfoque es el de Cambers, Carter, Wells, Bagwell, Padget y Thomson (2000), que afirman que es un proceso sofisticado que involucra habilidades cognitivas, como el 
análisis, la evaluación, las inferencias, la apertura a diversas afirmaciones o verdades universales. Para Giancardo (2001) es la capacidad del ser humano para resolver preguntas, asuntos y problemas que involucra diversas habilidades cognitivas, como la interpretación, el análisis, la evaluación, la inferencia, la explicación y la autorregulación del conocimiento. Y Santuiste (2001) señala que es un pensamiento reflexivo para responder de manera razonada y coherente a problemas y preguntas. (Alvarado, 2014, pp. 11,-12)

A su vez Glaser (1942) lo concibe como el esfuerzo para evaluar una creencia o forma de conocimiento a la luz de la evidencia que la soporta. De acuerdo con Treffinger, Isaksen y Dorval (2006), es la capacidad de afrontar cambios o problemas por medio de la organización y el análisis de posibilidades, la redefinición y el desarrollo de posibilidades promisorias, la jerarquización y prioridad de opciones para luego seleccionarlas. Facione (1990: 2) ofrece una definición muy completa al afirmar que el pensamiento crítico involucra juicios deliberados y autorregulados que devienen en interpretaciones, análisis, evaluaciones e inferencias, así como en consideraciones sobre aspectos contextuales, metodológicos y conceptuales relacionados con las evidencias en que se sustentan dichos juicios. (Alvarado, P. (2014, p. 13).

De estas estas definiciones y conceptualizaciones podemos concluir que los teóricos del pensamiento crítico toman como punto de partida la argumentación, que permite a los estudiantes formular y evaluar información relevante apoyada en evidencias y convertirse en personas autónomas y críticas ante el conocimiento.

Los autores Scriven, M. y R, Paul (2003) expresan que: “...el pensamiento crítico es el proceso intelectualmente disciplinado de conceptualizar, aplicar, analizar, sintetizar y/o evaluar, de manera activa y diestra, información reunida de, o generada por, la experiencia, reflexión, razonamiento o comunicación, como guía para la creencia y la acción" (p: 27). Según su visión, el pensamiento crítico tiene dos componentes: un conjunto de habilidades de generación y procesamiento de información y creencias, y el hábito de utilizar dichas habilidades mediante un compromiso intelectual. De esta forma, se entiende que el pensamiento crítico se opone a la adquisición y retención de información, ya que el pensamiento crítico implica una búsqueda activa de la información. Los autores aclaran también, que no es suficiente contar con la habilidad del pensamiento crítico, sino que la misma debe ser ejercitada.

Sin embargo, otras aportaciones al pensamiento crítico vienen de los referentes de la teoría positivista. Haskins (2006, p. 108) define el pensamiento 
crítico como "enseñar a los estudiantes a analizar y desarrollar tareas de lectura y escritura desde la perspectiva de los patrones formales y lógicos de coherencia." Aunque es importante lo que dicta esta definición, se entiende que contiene sus limitantes. El pensamiento crítico va más allá de pensar de forma lógica o analítica. Se trata de pensar de forma más racional y objetiva. Este es un punto importante ya que existe una gran diferencia en estas dos formas de pensar. La lógica y el análisis son conceptos filosóficos y matemáticos, mientras que pensar de manera racional y objetiva implica conceptos más amplios que tienen su origen en la psicología y sociología.

Los positivistas naturalmente se apoyan más en la lógica, dejando fuera algunas materias más amplias y subjetivas como lo son los estudios sociales, las lenguas y las artes. De todas estas referencias deducimos, que el pensamiento crítico debe formar parte integral de todas las asignaturas del currículum y debe ser un estándar o una competencia obligatoria a desarrollar en los alumnos en todos los niveles de proceso educativo.

En el lado opuesto a este enfoque positivista Facione (2007, pp. 4-68) propone seis habilidades cognitivas esenciales del pensamiento crítico: interpretación, análisis, evaluación, inferencia, explicación y auto regulación y propone siete disposiciones que llevan a la persona a enfocar el pensamiento crítico como un "estilo de vida": ser inquisitivo, juicioso, buscador de la verdad, confía en el razonamiento, sistemático, analítico y de mente abierta. La persona que practica el pensamiento crítico debe poseer dichas cualidades.

Por tanto no creo equivocarme al afirmar que los profesores universitarios están convencidos cada vez más de que los estudiantes necesitan ser formados para pensar críticamente, entendiendo esto como el modo de pensar sobre cualquier tema, contenido o problema junto con la aplicación de estándares intelectuales como la claridad, la exactitud, la precisión, la relevancia, la profundidad y la importancia que le servirán para evaluar sus pensamientos.

A continuación un documento presentado por Paul y Elder (2002, pp. 1013) con relación a los estándares intelectuales universales y su aplicación - sobre todo en algunos estados de los Estados Unidos-; estándares que podrían servirnos de referentes en la práctica y metodología del pensamiento crítico en la universidad. 


\section{TABLA NO. 1: PREGUNTAS QUE SE PUEDEN USAR PARA APLICAR LOS ESTÁNDARES INTELECTUALES UNIVERSALES}

\begin{tabular}{|c|c|}
\hline Claridad & $\begin{array}{l}\text { ¿Podría elaborar un poco más sobre ese punto? ¿Podría expresar ese punto } \\
\text { de otra manera? ¿Podría ilustrar el punto? Podría darme un ejemplo? }\end{array}$ \\
\hline Exactitud & $\begin{array}{l}\text { ¿Es eso cierto? ¿Cómo podríamos verificarlo? } \\
\text { ¿Cómo podríamos asegurarnos de que es verdad? }\end{array}$ \\
\hline Precisión & $\begin{array}{l}\text { ¿Podría dar más detalles? ¿Podría ser más específico? } \\
\text { ¿Podría precisar mejor? }\end{array}$ \\
\hline Pertinencia & $\begin{array}{l}\text { ¿Cómo se conecta esto con la pregunta? } \\
\text { ¿Qué tiene que ver con el tema? } \\
\text { ¿Cómo nos ayuda en el tema? }\end{array}$ \\
\hline Profundidad & $\begin{array}{l}\text { ¿Cómo enfoca o maneja la respuesta las complejidades de la pregunta? } \\
\text { ¿Cómo se tienen en cuenta los problemas que involucra la pregunta? } \\
\text { ¿Está atendiendo la pregunta los factores más significativos? }\end{array}$ \\
\hline Amplitud & $\begin{array}{l}\text { ¿Es necesario considerar otro punto de vista? } \\
\text { ¿Hay otra manera de enfocar este problema? } \\
\text { ¿Cómo podría mirarse esto desde una perspectiva conservadora? } \\
\text { ¿Cómo se vería esta situación o problema desde el punto de vista de....? }\end{array}$ \\
\hline Lógica & $\begin{array}{l}\text { ¿Es esto verdaderamente lógico? ¿Esto se desprende de lo que se dijo? } \\
\text { ¿De qué manera lo hace? } \\
\text { ¿Por qué antes la implicación era una y ahora parece ser otra? } \\
\text { ¿Cómo pueden las dos ser ciertas? }\end{array}$ \\
\hline Importancia & $\begin{array}{l}\text { ¿Es este el problema más importante que hay que considerar? } \\
\text { ¿Es esta la idea central en la que hay que enfocarse? } \\
\text { ¿Cuál de estos datos es el más importante? }\end{array}$ \\
\hline Imparcialidad & $\begin{array}{l}\text { ¿Tengo un interés personal en este asunto? } \\
\text { ¿Represento justamente los puntos de vista de otros? }\end{array}$ \\
\hline
\end{tabular}

Fuente: Paul y Elder (2002, pp. 10-13)

Los autores Paul y Elder (2002:22) enfatizan los estándares intelectuales que califican como los más significativos para el desarrollo del pensamiento crítico: "claridad, exactitud, precisión, pertinencia, profundidad, amplitud y lógica". Según los autores, "los estándares deben aplicarse a los elementos del pensamiento para luego convertirse en destrezas".

Los estándares intelectuales son pautas que le indican al docente qué deben aplicar los alumnos al pensamiento cada vez que se enfrente a un tema o problema que debe resolver. Las preguntas propuestas por Paul y Elder en la Tabla 1, se pueden usar para aplicar los estándares intelectuales universales facilitándole al docente la enseñanza de los mismos. La calidad del razonamiento del alumno dependerá del nivel en el que domine dichos estándares. 
La práctica de este ejercicio resultará en que el alumno interiorice este proceso y su razonamiento cada vez sea mejor.

\section{¿PERO QUÉ SUCEDE REALMENTE EN EL AULA UNIVERSITARIA?}

El predominio en la enseñanza tradicional, enfocada a la memorización de conocimientos, impide que el estudiante integre estos conocimientos a la experiencia diaria; el uso de técnicas expositivas propicia una actitud pasiva y receptora que inhibe una reflexión crítica en la construcción de sus aprendizajes. En este tipo de educación los puntos de vista de los estudiantes son considerados irrelevantes. Los estudiantes deben ser los protagonistas en el proceso de enseñanza-aprendizaje, contar con capacidad crítica para evaluar hechos, acciones y opiniones, por lo que las experiencias de aprendizaje que diseñen los docentes tendrán como objetivo la reflexión crítica del entorno exterior e interior del estudiante.

Pues es un hecho que la metodología utilizada por los profesores influye en los modos de aprender de sus alumnos; por ello los especialistas en pensamientos crítico recomiendan la aplicación de una metodología centrada en el aprendizaje y orientada a la solución de problemas en el aula se fomenta en los estudiantes el desarrollo del pensamiento crítico, la orientación a la producción y resolución de problemas y la toma de decisiones, volveremos sobre este punto más adelante.

\section{¿Y CÓMO DESARROLLAR EL PENSAMIENTO CRÍTICO EN ELAULA?: DOCENTE Y ESTRATEGIAS DIDÁCTICAS}

Generar en los estudiantes un pensamiento crítico como producto de un trabajo diario en el aula implica en primera instancia, que el profesor esté en conocimiento y convencido en qué consiste este tipo de pensamiento y sus implicaciones, si esto no se da ya es cuestión de ética docente. Reafirmemos que el pensamiento crítico es ante todo "una estrategia cognitiva, que se refiere al intento de los estudiantes de pensar de un modo más profundo, reflexivo y cuestionador el material de estudio" (Pintrich y García. 1993. Citado por Chiecher A.et al. (2006, p. 36). en acción:

Veamos algunos ejemplos de comportamientos que muestran esta estrategia

a) Invitar a los estudiantes a buscar evidencias cuando no se está convencido de un argumento,

b) Llevarlos a cuestionarse ente los planteamientos analizados.

c) Propiciar el desarrollo de puntos de vista personales a partir de las ideas expuestas en los textos.

d) Invitarlos a pensar ideas alternativas ante un determinado hecho. 
Según Paul. y Elder. (2003) se puede decir que una persona que piensa de forma crítica es aquella que posee:

- Autonomía: criterio para tomar decisiones por sí mismo sin dejarse manipular por lo que otros piensen o digan.

- Curiosidad: deseo y disposición para conocer la verdad y entender el mundo que le rodea.

- Coraje intelectual: capacidad para expresar y defender sus ideas, creencias, puntos de vista y ser capaz de mantenerlos a pesar de las oposiciones.

- Humildad intelectual: conocer sus límites y poderlos corregir.

- Empatía intelectual: capacidad para ponerse en lugar de otros para entenderlos.

- Integridad intelectual: reconocer la necesidad de la verdad en los valores, normas morales e intelectuales en los juicios de conducta o en puntos de vista de los otros.

- Perseverancia intelectual: disposición y necesidad de la verdad y del crecimiento intelectual a pesar de las dificultades.

- Tiene fe en la razón: desarrollo de sus facultades racionales, pensar coherente y lógicamente, persuadir a otros mediante razones y convertirse en personas razonables a pesar de los obstáculos. (p, 22)

De lo afirmado se sigue la importancia de que el profesor debe ser consciente que el pensamiento crítico es auto correctivo porque permite lanzarse en una búsqueda activa de sus propios errores, pensando en la autocorrección; de esta forma permite al alumno darse cuenta cuándo está en un error o cuándo su posición es impertinente. Según esta característica, si se quiere generar un pensamiento crítico en el aula de clase, ésta ha de convertirse en una comunidad indagadora e inquisidora, donde los estudiantes empiezan a darse cuenta y a corregir los métodos y procedimientos usados por los compañeros, así como los propios; por ello son capaces de auto corregirse.

Por su lado Páez H. (1992), considera tener en cuenta de manera especial la característica de la contextualidad, es decir, la sensibilidad al contexto es otra característica importante que permite desarrollar un pensamiento flexible que posibilite reconocer cómo los diversos contextos requieren diversas aplicaciones de reglas y de principios, esto posibilita que las áreas curriculares, por ejemplo, de sociales y filosofía sean áreas pertinentes para desarrollar el pensamiento crítico de los estudiantes. Pues a través del uso de estrategias de enseñanza y de aprendizaje adecuadas ayudan a los estudiantes a tener buen juicio sobre lo estudiado: a hacer una buena interpretación de un texto escrito, a redactar una composición coherente, a comprender lo que escuchan en la clase, a proporcionar sólidos argumentos al 
asumir una posición, a detectar las posiciones asumidas, sugeridas o implícitas por un autor. Estas destrezas de razonamiento posibilitan hacer inferencias, indagar, formarse sus propios conceptos y transferir el conocimiento al contexto particular del estudiante. (p. 77)

Asimismo, Paul y Elder (2003), enfatizan en que desarrollar el pensamiento crítico es fundamental en un proceso enseñanza aprendizaje ya que permite al estudiante:

- Formular preguntas vitales y problemas dentro de éstas, enunciándolas de forma clara y precisa.

- Poder recopilar y evaluar la información recibida, usando ideas para interpretarla con perspicacia y aplicándola a su propia vida.

- Generar, formular e inferir conclusiones y soluciones para no quedarse con los conceptos mínimos; por el contrario, manejarlos, ampliarlos y aplicarlos.

- Ser capaz de adoptar un punto de vista determinado, reconociendo $\mathrm{y}$ evaluando según sea necesario, sus supuestos, implicaciones y consecuencias.

- Aprender a comunicarse de una forma lógica y fundamentada expresando sus ideas y entrando en diálogo con los demás para enriquecer su concepción de la realidad.

- Poder relacionar los contenidos de una asignatura con los de otras y con lo que es importante en la vida. (p. 30).

\section{EL ENFOQUE PROBLEMATIZADOR}

Lynch y Wolcott (2001) afirman, que muchas de las tareas que encargamos a nuestros estudiantes tienen que ver con repetir o parafrasear la información que les transmitimos, pero éste es sólo el primer paso para desarrollar un pensamiento crítico. Es muy importante trascender este nivel y aprender a cuestionar los datos. El enfoque general de la enseñanza debe ser problematizador: hacer hincapié en las incertidumbres y el cuestionamiento a partir de la información recabada.

Nos dicen los autores que estrategias como el método de proyectos, el estudio de casos o la solución de dilemas morales son muy recomendables para favorecer el pensamiento crítico si el profesor sabe dirigir debates y cuestionar oportunamente la argumentación de sus estudiantes. Desde este enfoque, Lynch y Wolcott proponen varias recomendaciones para desarrollar el pensamiento crítico en los estudiantes:

1. Dada una situación, pedirles que identifiquen el problema, la información relevante y las incertidumbres. 
2. Explorar la interpretación y las conexiones: implica interpretar la información identificando los aspectos relevantes y controlando los propios prejuicios y organizando los datos de manera significativa para buscar una solución.

3. Pedirles que prioricen las alternativas de posible solución y comunicarlas.

4. Solicitarles que integren, monitoreen y refinen las estrategias para abordar el problema. Implica dar cuenta de las limitaciones de la solución que ellos han propuesto. Se recomienda empezar por estructurar las tareas y las discusiones de clase para favorecer las habilidades de pensamiento crítico desde un nivel elemental y paulatinamente ir desarrollando habilidades de investigación hasta que los estudiantes sean capaces de proponer soluciones alternativas a los problemas. Existen numerosos recursos didácticos en Internet y en los libros dedicados al tema de los que se puede echar mano para ayudar a los estudiantes a convertirse en pensadores más refinados y críticos. (Lynch y Wolcott, 2001).

\section{LA PREGUNTA Y EL FORO VIRTUAL COMO ESTRATEGIA DIDÁCTICA}

Villegas V, et. al (2005: 45) plantean como estrategia importante para el desarrollo del pensamiento crítico el uso de la pregunta en clase; según este planteamiento, la formación de un pensamiento crítico implica la oferta de oportunidades para que el alumno aprenda a indagar y a cuestionar y la pregunta es un buen recurso didáctico. Queremos decir que las actividades enseñanza-aprendizaje deben orientarse hacia las preguntas y problemas mediante la conversación, la discusión y la investigación se intentarán construir las respuestas y las soluciones. Según el autor, preguntar, conversar, leer y escribir son actividades fundamentales en el aprendizaje de una ciencia humana. Aprender ciencias humanas supone aprender a preguntar, hablar, leer y escribir sobre las mismas.

Por su lado Fëdorov, A. (2006) propone que el desarrollo del pensamiento crítico, concebido como un pensamiento de calidad, puede llevarse a cabo por medio de los foros. Indica, apoyado en varios autores, que los foros, sobre todo los virtuales, son excelentes estrategias para el desarrollo del pensamiento crítico porque involucran múltiples aspectos cognitivos y socioafectivos, como seguir el hilo de los diálogos, pensar y entender las intervenciones, confeccionar mensajes para impulsar el diálogo hacia delante, dejar "volar" la expresión de los demás, respetando la autonomía de los participantes y salir de lo evidente para explorar diferentes alternativas, entre otras muchas posibilidades. 
Una alternativa como el foro virtual constituye una herramienta tecnológica que, a través de una metodología adecuada propicia la creación de los ambientes que estimulan el aprendizaje y el pensamiento crítico a través de la participación, la negociación y construcción del conocimiento. Por tanto en nuestra Casa de estudios deberíamos fomentar el uso de esta herramienta virtual.

Para fomentar el pensamiento crítico Rolón ( 2014) recomienda:

1. Crear ambientes pedagógicos que posibiliten a los estudiantes ejercitarse en el diálogo y la participación, de modo tal que conviertan el aula en una comunidad de investigación.

2. Buscar la coherencia entre los objetivos del curso, las actividades de aprendizaje y los procedimientos de evaluación.

3. Utilizar las repuestas de los estudiantes como elementos diagnósticos de la profundidad de su pensamiento y de las estrategias que emplean para argumentar.

4. Es permitente analizar la relevancia, confiabilidad y fuerza de los argumentos que emplean los estudiantes.

\section{EL PENSAMIENTO CRÍTICO EN LA UNIVERSIDAD, SUS PRINCIPALES ELEMENTOS}

Creo que todos convenimos en que uno de los objetivos de la Universidad de los tiempos actuales es formar personas y profesionistas creativos, críticos y capaces de contribuir a resolver los problemas que aquejan a la sociedad, por lo que es muy importante que en su formación los estudiantes tengan la oportunidad de ejercitar y desarrollar el pensamiento crítico. Para hacerlo posible, los docentes deben diseñar estrategias didácticas para que los estudiantes participen de manera activa y ejerciten realmente sus habilidades

De lo afirmado se infiere, que buscamos crear en los futuros profesionales un perfil que les permita desarrollar no sólo las habilidades de su profesión, sino también formarse como seres humanos integrales. El perfil de nuestros egresados está basado en las competencias, por lo que necesitamos cultivar una serie de habilidades y capacidades que les permitan un desarrollo integral y un crecimiento permanente. Para Rolón (2014), algunas de las habilidades del pensamiento crítico que se deben desarrollar son las siguientes:

- Identificar conceptos o ideas centrales.

- Reconocer suposiciones subyacentes.

- Evaluar la evidencia en que se basan los argumentos. 
- Buscar datos adecuados.

- Verificar hechos.

- Buscar información.

- Tomar decisiones.

- Dejar de lado los prejuicios.

A lo que suma Marzano (2005), quien agrega:

- Ser preciso y buscar precisión.

- Ser claro y buscar claridad.

- Mantener la mente abierta.

- Refrenar la impulsividad.

- Adoptar una postura cuando la situación lo exija.

- Responder de manera apropiada a los sentimientos y al nivel de conocimiento de los demás.

Sigue afirmando Rolón (Ibid), que buscamos, además, un desarrollo integral de las capacidades y la apropiación de valores que ayuden al estudiante a ubicarse en su vida personal y profesional. El pensamiento crítico en la universidad nos permite sugerir al estudiante entrar en contacto con un saber profundo. Esto es, que su saber no se quede en una sola manera de ver lo ya establecido, ya que siempre existe algo más. Se le debe enseñar a cuestionar y, a partir de ahí, comenzar un proceso de aprendizaje profundo que derive en un aprendizaje significativo.

En síntesis, el pensamiento crítico en la universidad nos permite formar jóvenes competentes e inconformes con su propio saber, y de ahí derivar la búsqueda permanente de alternativas para solucionar problemas concretos. Con la promoción del pensamiento crítico aspiramos también a formar jóvenes con perspectivas de cambio y herramientas para la búsqueda del mismo. Nos permite egresar profesionales permanentemente inconformes con lo establecido y con la capacidad de dar respuestas a demandas concretas con argumentos que les permitan sustentarlas.

Un pensamiento crítico en la universidad nos permitiría formar expertos competentes no sólo en su quehacer profesional, sino también en la vida.

\section{LAFORMACIÓN DEL PENSAMIENTOCRÍTICOYLASCOMPETENCIAS CÍVICAS, ÉTICAS Y CIENTÍFICAS}

En este apartado intento presentar otro enfoque acerca del pensamiento crítico diferente a las de las capacidades reflexivo- cognitivas que hemos 
desarrollado en apartado anteriores; pues la formación del pensamiento crítico se está retomando con fuerza en las agendas educativas debido a que se encuentra estrechamente relacionada con algunos de los principales objetivos de las reformas educativas actuales, tales como la formación de competencias cívicas y éticas y el desarrollo del pensamiento científico. Pues el desarrollo del pensamiento crítico es la base de la preparación para que los sujetos aprendan a cuestionar y preguntarse el porqué de las cosas, actitudes que se encuentran detrás de la búsqueda científica y filosófica sobre el mundo.

Por otro lado, en las sociedades del conocimiento el acceso a los discursos, saberes y teorías de las diferentes profesiones pasa necesariamente por el acceso a las ciencias vinculadas con dichos conocimientos, pues todas las profesiones de hoy se sustentan en y mediante la aplicación del saber científico. Por lo que el acceso al pensamiento científico y los procesos para favorecerlo son cuestiones indispensables para la dotación de oportunidades para las nuevas generaciones.

También la formación del pensamiento crítico está vinculada con la creación de capacidades para el aprendizaje permanente, la investigación, la innovación y la creatividad. Ya que genera mentes activas y científicas, habituando a los alumnos en el ejercicio del razonamiento, el pensamiento lógico, la detección de falacias, la curiosidad intelectual y por el saber y la solución de problemas. Forma capacidades para la duda y el cuestionamiento permanente con base en argumentos y razones fundamentadas científicamente (Patiño, 2012, p. 89).

En este marco, la formación del pensamiento crítico apunta también hacia la formación de ciudadanos autónomos capaces de actuar y tomar decisiones con criterio propio, aspectos necesarios para la vida profesional y ciudadana en nuestras sociedades.

En términos de creación de competencias cívicas y éticas, la formación del pensamiento crítico es un asunto central en la educación de ciudadanos activos y con criterio propio, indispensable para una toma de decisiones autónoma y responsable de los ciudadanos en relación con sus propias vidas y sobre las de otras personas en contextos democráticos. En este sentido, por un lado, es un ingrediente imprescindible de la autoconstrucción de la persona, ya que le brinda capacidades para la independencia, tomar distancia de las injusticias, formar el propio criterio, conocerse y autoexaminarse a sí mismo y contribuir a la formación de su autonomía.

Por otro lado, contribuye a generar capacidades para la comprensión y el entendimiento mutuo entre las personas, la convivencia en la diversidad y la 
posibilidad de llegar a acuerdos y entendimientos entre ciudadanos que piensan diferente, al enseñar a mirar desde la perspectiva de otras personas. Enseñar a conocerse y examinarse a uno mismo y tomar en serio el punto de vista de los demás contribuye a la formación en la convivencia democrática, la tolerancia y la resolución pacífica de los conflictos (Nussbaum, 2010, p. 82).

Todas estas competencias éticas y ciudadanas son muy importantes para que las personas sean capaces de ejercer su libertad con responsabilidad y se comprometan con un mundo mejor, a través del diálogo, la deliberación y la colaboración.

\section{A MANERA DE CIERRE}

Desarrollar el pensamiento crítico se plantea como un reto para la educación actual en todos los niveles y muy en especial en la universidad ante los cambios y exigencias que presenta el mundo contemporáneo.

El desarrollo teórico y práctico del pensamiento crítico debe ser una responsabilidad curricular en la universidad. No podemos esperar que de forma intrínseca esta habilidad sea desarrollada en las instituciones educativas. El pensamiento crítico-reflexivo debe ser un producto del currículum. Su inserción en el plan de estudios de toda institución educativa debe ser un ejercicio calculado y acertado. Debe ser comprendido por todos los actores del proceso curricular y también debe ser evaluado.

El desarrollo del pensamiento crítico es una necesidad humana y social, ya que una persona que no posee pensamiento crítico no tendrá la misma calidad de vida que aquella que si lo posee. El pensamiento crítico va más allá del aula de clase, se aplica a todas las actividades cognitivas de la vida diaria, como ser: las decisiones que toma una persona, los argumentos que fórmula y las concepciones que forma del mundo y las personas.

La formación del pensamiento crítico está vinculada con la creación de capacidades para el aprendizaje permanente, la investigación, la innovación y la creatividad. Genera mentes activas y científicas, habituando a los alumnos en el ejercicio del razonamiento, el pensamiento lógico, la detección de falacias, la curiosidad intelectual y por el saber y la solución de problemas. Forma capacidades para la duda y el cuestionamiento permanente con base en argumentos y razones fundamentadas científicamente. 
En términos de creación de competencias cívicas y éticas, la formación del pensamiento crítico es un asunto central en la educación de ciudadanos activos y con criterio propio, indispensable para una toma de decisiones autónoma y responsable de los ciudadanos en relación con sus propias vidas y sobre las de otras personas en contextos democráticos. En este sentido, es un ingrediente imprescindible de la autoconstrucción de la persona, ya que le brinda capacidades para la independencia, tomar distancia de las injusticias, formar el propio criterio, conocerse y autoexaminarse a sí mismo y contribuir a la formación de su autonomía (Patiño, 2010).

\section{REFERENCIAS BIBLIOGRÁFICAS}

Alvarado, P. (2014) "El desarrollo del pensamiento crítico: una necesidad en la formación de los estudiantes universitarios" DIDAC Nueva Época, No. 64julio-diciembre 2014 - Universidad Iberoamericana.

Facione, P. (2007). Pensamiento Crítico: ¿Qué es y por qué es importante?

Recuperado el 22 de abril de 2007, de www.eduteka.org/

PensamientoCriticoFacione.php

Facione, P. (1990). Pensamiento Crítico: Una Declaración de Consenso de Expertos con Fines de Evaluación e Instrucción Educativa. Recuperado el 20 de abril de 2007, de http://www.insightassessment.com/articles.html

Fëdorov A. (2006). Siglo XXI, la universidad, el pensamiento crítico y el foro virtual. Centro de Desarrollo Académico del Instituto Tecnológico de Costa Rica. En: Revista Iberoamericana de Educación. OEI. MEN. Comisión Nacional para el Desarrollo de la Educación Superior. Febrero, Bogotá. 1997, p.15. Disponible en: (http://www.rieoei.org/deloslectores/1218Federov.pdf.)

Haskins, G.R. (2006). A Practical Guide to Critical Thinking. Recuperado el 21 de abril de 2008, de www.skepdic.com/essays/haskins.pdf

Lynch, C. \& Wolcott, S. (2001). Helping your students develop critical thinking skills, IDEA Paper No. 37, http://www.theideacenter.org/sites/default/files/ IDEA_Paper_37.pdf. Consultado: Abril, 2015. 
Marzano, R. J. (2005). “Dimensiones del aprendizaje”, En: Iteso. No. 11: Octubre-Diciembre. México- Disponible en: http://legisweb.state.wy.us/ InterimCommittee/2012/Z02MarzanoLevels.pdf

Nussbaum, M. (2010). Why Democracy Needs the Humanities. Princeton University Press, On line: http://democracyeducationjournal.org/cgi/ viewcontent.cgi? article $=1028 \&$ context $=$ home

Páez H. (1992). El desarrollo del pensamiento crítico del estudiante. Una finalidad educativa pendiente del área curricular de Estudios Sociales. México: Gidesa

Patiño, H. (2012). "El pensamiento crítico como tarea central de la educación humanista”. DIDAC Nueva Época, No. 60- julio-diciembre 2012 Universidad Iberoamericana-

Paul, R. y Elder, L. (2002). La mini guía para el pensamiento crítico. Conceptos y herramientas. Recuperado el 25 de agosto de 2016, de http://www.criticalthinking.org/resources/PDF/SP-ConceptsandTools.pdf

Paul, R. y Elder L. (2003). Bolsilibro para estudiantes sobre cómo estudiar y aprender. Fundación para el Pensamiento Crítico. Disponible en internet en: www. criticalthinking.org] consultado el 24 de Julio de 2016.

Rolón, N. (2014). “Pensamiento crítico y docencia. Breves reflexiones de su aporte y riqueza".

DIDAC Nueva Época, No. 64- julio-diciembre 2014 - Universidad IberoamericanaScriven, M. y R. Paul, 2003. Defining Critical Thinking.

En: www.hennievandyk.bravepages.com/thinking.html. Visitado en 2 de Marzo, 2015.

Villegas Valero et al. (2005). "Las preguntas en la enseñanza de las ciencias humanas", OEI. En: Revista Iberoamericana de Educación, No. 8. 